\title{
LA SEMIÓTICA EN VENEZUELA. HISTORIA, SITUACIÓN ACTUAL Y PERSPECTIVAS
}

\author{
José Enrique Finol y Dobrila Djukich 1
}

Presidente de la Asociación Venezolana de Semiótica (AVS)

Laboratorio de Investigaciones Semióticas y Antropológicas

Universidad del Zulia (Maracaibo, Venezuela) ${ }^{2}$

\section{INTRODUCCIÓN}

Los inicios de la enseñanza y la investigación semiótica en Venezuela están temporalmente ubicados en la década de los sesenta y culturalmente orientados por la presencia e influencia de la tradición europea, particularmente francesa, en los medios intelectuales y uni-

1 Agradecimientos. El autor desea expresar su agradecimiento a Rocco Mangieri, Teresa Espar, Víctor Fuenmayor y Frank Baiz por la información aportada para la elaboración del presente artículo. Asimismo, un agradecimiento especial a Dobrila Djukich de Neri por la revisión del manuscrito y por sus acertadas sugerencias.

${ }_{2}$ Universidad del Zulia, Facultad de Ciencias, Laboratorio de Investigaciones Semióticas y Antropológicas. Avenida Universidad, Edificio Grano de Oro. Maracaibo, Venezuela. E-mail: jefinol@ven.net y también jefinol@solidos.ciens.luz.ve. 
versitarios del país. En las ciencias y la cultura, Venezuela ha sufrido dos influencias importantes y, en cierto modo, complementarias. Por un lado, la presencia desde comienzos de siglo de las compañías petroleras influyó determinantemente en la orientación de los estudios de orden científico y tecnológico en Venezuela. La condición de país petrolero, producto del descubrimiento en 1920 del llamado oro negro, hizo que desde temprano la ciencia y la tecnología, así como los estudios en ciencias de la salud, estuviesen vinculados a centros universitarios de los Estados Unidos, país al que fueron enviados numerosos jóvenes venezolanos a formarse y de donde vinieron ingenieros y técnicos para ocupar las posiciones más relevantes en las compañías internacionales que explotaban el petróleo.

Por otro lado, desde el punto de vista de las ciencias sociales y humanas y de lo que tradicionalmente se llama la cultura, incluidas las bellas artes, la música y la literatura, la vinculación más activa y profunda fue con Europa, en particular con España y Francia. La mayoría de nuestros más importantes pintores, escritores, escultores, músicos y poetas tenían en sus planes la pasantía obligatoria por París o Madrid y, ocasionalmente, también por Roma. Asimismo, nuestros estudiosos de filosofía, literatura, educación y otras ramas afines consideraban a Europa el lugar ideal para continuar su formación intelectual. Incluso uno de nuestros más conspicuos dictadores, el general Guzmán Blanco, que dirigió al país durante el período conocido como «el Despotismo Ilustrado» (1870-1877 y 1879-1884), tenía como objetivo de gobierno «convertir a Caracas en un pequeño París», y su «labor progresista cultural (se proponía) colocar a Venezuela a imagen y semejanza de Francia» (Rodríguez, 1988: 392-93).

A partir de esa vinculación con autores y escuelas europeas, la semiótica en Venezuela ha conocido un constante desarrollo de publicaciones y de programas de enseñanza que han permitido hacer una contribución significativa en el mundo semiótico latinoamericano. Igualmente, la semiótica ha hecho contribuciones importantes como disciplina aplicada, en particular en las áreas de la publicidad ${ }^{3}$, de la danza ${ }^{4}$ y de la televisión ${ }^{5}$.

3 En el área de la publicidad son notables los trabajos de Julián Cabeza Lora.

4 En la danza, y también en la literatura, son notables los trabajos de Víctor Fuenmayor.

5 En el área de los medios de difusión masiva son notables los análisis, para una empresa de televisión, hechos por el profesor Manuel Bermúdez, en Caracas, cuyo énfasis fundamental fue la telenovela venezolana. 


\section{LA PRIMERA GENERACIÓN DE SEMIÓTICOS VENEZOLANOS 6}

La Semiótica, pues, llega a Venezuela, como era de esperarse en el contexto antes indicado, de la mano de los llamados estudios literarios franceses y de la linguiística europea, y éstos, a su vez, de la de los jóvenes profesores universitarios que fueron a París a completar su formación y a buscar nuevas orientaciones y conocimientos que contribuyeran a cambiar la aburrida enseñanza y escasa investigación de la literatura, hasta entonces sumida en los análisis impresionistas, hiper subjetivos y a veces fantasiosos que conducían a los estudiantes al más absoluto fastidio intelectual o a la más azucarada melancolía. También profesores venidos de España a las universidades venezolanas trajeron a las escuelas de letras, periodismo, educación, antropología, sociología y filosofía los trabajos de Ferdinand de Saussure, Roland Barthes, Algirdas J. Greimas, Lucien Goldmann, Julia Kristeva, Claude LéviStrauss, Umberto Eco, Tzvetan Todorov, Christian Metz, Ferrucio Rosi-Landi y tantos otros. No será sino a principio de la década de los noventa cuando la Semiótica norteamericana, basada en los trabajos de Charles Sanders Peirce, llegará por primera vez a Venezuela.

La lingüística saussureana llegó a Venezuela, como a todos los países latinoamericanos, gracias a la maravillosa traducción del Curso de Lingüistica General, hecha por Amado Alonso y publicada por la Editorial Losada, S.A., de Argentina traducción que había aparecido por primera vez en el mundo hispánico en $1945^{7}$. Junto con la linguística y los estudios literarios se asomó por primera vez a nuestras aulas de clase, lo que inicialmente se definía como «una ciencia que estudie la vida de los signos en el seno de la vida social» (Saussure, 1965: 60). Esa nueva disciplina, surgida en medio del positivismo europeo, subyugó a todos por igual: escritores, sociólogos, críticos literarios, lingüistas, filósofos, antropólogos; igual a estudiantes que a profesores, a izquierdistas y derechistas, a marxistas y liberales. Todos

6 Como toda división temporal la presente clasificación en «generaciones» es arbitraria. Se trata de un intento de ubicar, según ciertos parámetros de tiempo, los inicios y la continuidad de la enseñanza y la investigación semiótica en el país.

7 Sucesivas ediciones hechas por la misma editorial aparecieron en 1955, 1959, 1961,1965 . Nótese que entre la primera y segunda edición transcurren diez años, mientras que en las subsiguientes tal período se reduce a cuatro años, luego a dos, luego a cuatro, lo que parecería indicar la creciente popularidad de uno de los libros que más ha influido en las ciencias humanas y sociales de este siglo. 
reivindicaban la nueva disciplina como congruente, en sus principios $\mathrm{y}$ aplicaciones, con lo que cada cual estudiaba.

De ahí que la Semiología adquiriría, junto con el estructuralismo en pleno apogeo, status universitario oficial, se convertiría en una disciplina de prestigio, y ya en los años setenta era casi masiva, por lo menos en aquellos escenarios universitarios que le eran propios o afines. Las librerías de ciudades como Caracas, Maracaibo, Mérida y Barquisimeto se llenarían de las más recientes traducciones de los libros recién aparecidos en Francia, Italia o España.

Los trabajos fundamentales de esta primera generación de semióticos venezolanos, entre quienes es importante mencionar a Víctor Fuemayor, José Pascual Buxó, Ana Mireya Uzcátegui, Roque Carrión Wam, Juan Gregorio Rodríguez Sánchez, Andrés García, Enrique Arenas, J.M. Delgado Ocando, Manuel Bermúdez, Oscar Moraña, Hugo McCormick, J. Pérez O’Higgins y Enrique Obediente, contribuyeron a la divulgación y a despertar el interés por la Semiótica entre académicos de las más distintas áreas científicas ${ }^{8}$.

En su gran mayoría, todos estos fundadores de la Semiótica en Venezuela se formaron al calor de las teorías europeas, en especial fueron determinantes los trabajos de Roland Barthes, Julia Kristeva, Umberto Eco y, un poco más tarde, de A.J. Greimas. Eran los tiempos del apogeo de la revista Communications, publicada por el Centre d'Études de Communications des Masses; eran los tiempos de los Elementos de Semiología, de Barthes, que tanta influencia tuvieron en la década de los sesenta y setenta. Los principales trabajos de investigación, en su casi totalidad, pertenecen, en esta época, al área de la semiótica literaria y al análisis de textos. Entre ellos destacan los análisis que el Dr. Víctor Fuenmayor hiciera de las principales novelas de los escritores venezolanos Rómulo Gallegos y Teresa de la Parra. En el área de la Semiótica Jurídica es necesario señalar los nombres de Roque Carrión Wam, fundador de esa área en Venezuela, y de J. M. Delgado Ocando; y en la del audiovisual fueron pioneros Manuel Bermúdez y Óscar Moraña. También en esta época estuvieron en Venezuela los psicoanalistas argentinos Hugo Bleichmar y Emilia de Bleichmar, quienes durante algunos años fomentaron, junto a Hugo McCormick, las investigaciones psicosemióticas.

8 En Mérida, a principios de la década del setenta, dictó un curso de semiótica Enrique Ballón, ex alumno de Greimas. 
En cuanto a la enseñanza, la Semiótica encuentra como escenarios privilegiados las escuelas de Letras, Comunicación Social, Arquitectura, Sociología y Antropología, a nivel de licenciatura en las distintas universidades del país. Muchos de los estudiantes de esas escuelas constituirán lo que, muy arbitrariamente y sólo con el propósito de establecer algunos parámetros mínimos de inteligibilidad, hemos llamado la segunda generación.

\section{LA SEGUNDA GENERACIÓN}

\subsection{De la Semiología a la Semiótica}

A finales de la década del ochenta la Semiótica conoció en Venezuela, como en casi todas partes del mundo, un declive. Cesó el efecto de la moda intelectual, se decantaron métodos y teorías y abandonaron el campo numerosos estudiosos e investigadores que hasta entonces estuvieron deslumbrados por el llamado boom de la Linguiística y de la Semiología, a las que no le veían futuro duradero, en particular a esta última. Para entonces, ya la joven disciplina, en el ámbito internacional, había aceptado como nombre único el de «Semiótica», un nombre más vinculado a la primera tradición de la filosofía griega y que la pujante escuela norteamericana, siguiendo a Charles Sanders Peirce (1839-1914), había tomado. La fundación de la Asociación Internacional de Semiótica, en 1969, marca en forma definitiva la declinación del nombre «Semiología» y la aceptación académica y oficial de la denominación «Semiótica».

\subsection{La Asociación Venezolana de Semiótica y su primer congreso nacional}

En 1989 los alumnos de los primeros semióticos venezolanos fundan la Asociación Venezolana de Semiótica, cuya directiva presidía el Dr. Víctor Fuenmayor, un ex alumno de Roland Barthes y Julia Kristeva, y que además integraban Iván Ávila, Ana Mireya Uzcátegui, 
Ana Ferrer, Rolando Navarro, Doris Pachano, María García y quien esto escribe. La convocatoria para la constitución de la asociación la firman Julián Cabeza, Iván Ávila y José Enrique Finol, y allí se indica que uno de los objetivos es «mancomunar esfuerzos para desarrollar una serie de actividades tendentes a difundir los avances de esta disciplina y las investigaciones de sus integrantes. Nuestras discusiones se realizarán obviamente en nuestro país pero tendrán también un alcance internacional, especialmente en América Latina». Igualmente se menciona que el día 15 de julio, en el marco del VII Encuentro Nacional de Docentes e Investigadores de la Lingüística, se había realizado una reunión preliminar «en la cual se designó un Comité Organizador». La reunión donde se funda la asociación se realiza en el Departamento de Ciencias Humanas de la Facultad de Ciencias de la Universidad del Zulia, en Maracaibo, y se registra en la misma ciudad como asociación civil sin fines de lucro el día 24 de abril de 1989.

Esa primera directiva, bajo el eslogan «Conciencia semiótica y lenguajes», organizará el I Encuentro Venezolano de Semiótica, el cual se realiza del 24 al 28 de abril de 1989 en los locales de la antes mencionada Facultad de Ciencias. Este primer congreso reúne a invitados de casi todo el país y el programa incluye ponencias, conferencias plenarias, cursos y seminarios.

Tanto la enseñanza como diversos trabajos de investigación continuaron durante este período. La mayoría de los miembros de esta generación, como sus mentores, se formaron en las escuelas europeas. Tal es el caso de Teresa Espar, Iván Ávila, Amado Durán, Liddis Palomares, Dobrila Djukich de Neri y José Enrique Finol, todos ex alumnos del Groupe de Recherches Sémio-Linguistiques, de L'École de Hautes Études en Sciences Sociales, en Francia, grupo dirigido por A.J. Greimas y J. Courtés, y que algunos autores han llamado la «Escuela de París» (Coquet, 1982). La mayoría de ellos apuntaron sus investigaciones hacia la Semiótica del Texto, en particular del texto literario, con la excepción de Ávila (semiótica del azar) y Finol (Semiótica del Mito). Otros, como Andrés García y Rocco Mangieri, se formaron bajo la dirección de Umberto Eco, en Bolonia, Italia, y Marinés Mendoza, en la Universidad Complutense de Madrid, España, bajo la dirección de Cristina Peña Marín. Asimismo, Julián Cabeza y Lourdes de Cabeza, ex alumnos de Bernard Pottier, también se formaron en Semiótica en París. Vale la pena mencionar que Andrés García Ildarraz fue quien introdujo en Venezuela los primeros análisis del objeto y del espacio desde un punto de vista semiótico. Últimamente 
sus trabajos han estado orientados hacia una semiótica del diseño. Otro de los miembros de esta generación que ha tenido una importante producción en semiótica literaria, en general orientada por las teorías de Julia Kristeva, ha sido Douglas Bohórquez. Igualmente, Vícto Bravo ha sido uno de los jóvenes de mayor productividad en este campo y en el de la crítica literaria. En esta época vale la pena mencionar la presencia en la Universidad del Zulia del Dr. José Sazbón, autor de numerosos trabajos sobre la semiótica y el estructuralismo, asesor de importantes editoriales en el ámbito latinoamericano y quien desempeñó una importante actividad académica durante su estadía de casi cinco años en Venezuela.

En la capital venezolana son conocidos los nombres, entre otros, de Jean-Louis Rebillou, quien trabaja en sociosemiótica y discurso político, Atilio Romero en arquitectura y urbanismo, y Frank Baiz, especialista en la semiótica greimasiana y en la narratología e interesado en problemas de la narración audiovisual y en el uso de instrumentos semióticos en el diseño. También trabajan en Caracas, Mireya Fernández, quien utiliza herramientas semióticas en sus investigaciones sobre competencia de la lectura, y Rosario de León, en literatura comparada. Asimismo, hay varios trabajos de François Ambard, sobre semiótica de las pasiones, y de Aquiles Estée, formado en la orientación peirceana y que se ha interesado en problemas relacionados con las nuevas tecnologías de la comunicación con un enfoque que las examina como integrantes de una compleja «ecología» cuyo impacto es global.

También en esta época jugó un papel estimulante el Centro Internacional de Semiótica y Linguíística de la Universidad de Urbino, en Italia, lugar a cuyos cursos y simposia de verano asistieron en ocasiones varios de los investigadores de esta generación, gracias en particular al estímulo del profesor Andrés García Ildarraz. Asimismo, los conocidos Documenti di Lavoro publicados por ese centro alimentaron las investigaciones en Venezuela.

Lamentablemente, ni la Asociación Venezolana de Semiótica ni sus congresos bienales tuvieron la continuidad esperada. Pese a ello, la actividad de enseñanza e investigación continuó con grupos activos en las ciudades de Mérida, Maracaibo, Caracas, Valencia y Barquisimeto. Actividades localizadas se dieron siempre y, en algunos casos, actividades nacionales que han contribuido a mantener productiva la actividad semiótica en la geografía académica venezolana. 
Bajo la coordinación del profesor Rocco Mangieri se creó en 1994 el Programa de Encuentros Internacionales de Semiótica, el cual organizó la venida a Venezuela de renombrados semióticos, entre ellos Umberto Eco, quien visitó el país del 27 de junio al 5 de julio de 1994, e hizo un recorrido por Caracas, Maracaibo, Mérida y Barquisimeto. Asimismo, el conocido semiótico italiano Paolo Fabbri visitó Venezuela en 1995. También Eric Landowski y Jean Marie Floch visitaron Mérida, Maracaibo y Caracas y han mantenido un contacto constante con los investigadores venezolanos. Más recientemente, en enero de 1998, vino a Mérida el conocido semiótico italiano Omar Calabrese. Actualmente se programa la visita de Francisco Vicente Gómez, de España. El objetivo fundamental del Programa de Encuentros Internacionales de Semiótica es el de «actualizar y perfeccionar la disciplina en el ámbito de las universidades y centros de investigación» ${ }^{9}$.

\subsection{El II Congreso Venezolano de Semiótica y los nuevos proyectos}

En 1997, del 21 al 26 de abril, por iniciativa de José Enrique Finol, se organizó el II Congreso Venezolano de Semiótica, también en Maracaibo, bajo el lema de «Confrontaciones, Integraciones y Pasiones Semióticas», encuentro que entre otras cosas se proponía reagrupar a los semióticos venezolanos, reactivar la Asociación Venezolana de Semiótica (AVS) y dar a conocer los trabajos de investigación y programas de enseñanza que se venían desarrollando en los últimos años. El comité organizador dirigido por América Rincón logró reunir a un importante grupo de investigadores de Venezuela, más seis invitados de otros países, quienes presentaron ponencias, dirigieron seminarios y cursos que han motivado a un importante grupo de jóvenes interesados en formarse en la disciplina en cuestión.

El día 26 de abril de ese mismo año se realizó la asamblea general de los miembros de la AVS y se designó una nueva Junta Directiva presidida por J. E. Finol e integrada además por Nancy Torres, Marinés Mendoza, Dobrila de Neri, Mariluz Domínguez e Iván Ávila. Allí

9 Rocco Mangieri en comunicación personal. 
se escogió la Universidad Central de Venezuela, en Caracas, como sede del tercer congreso a efectuarse en mayo de 1999. El Coordinador del Comité Organizador es el profesor Jean-Louis Rebillou, de la Universidad Central de Venezuela.

\subsection{La Escuela Latinoamericana de Semiótica (ELSE)}

La AVS, además de darle regularidad a sus reuniones científicas bianuales, aprobó en 1997 desarrollar dos iniciativas de vocación latinoamericana, con apoyo de la Universidad del Zulia, del Consejo Nacional de Investigaciones Científicas y Tecnológicas (CONICIT), y de la Fundación para el Desarrollo de la Ciencia y la Tecnología del Estado Zulia (FUNDACITE-Zulia). La primera fue la creación de la Escuela Latinoamericana de Semiótica (ELSE), concebida como escuela de verano, con la cual se busca, sobre todo, la formación y actualización de investigadores en las distintas áreas de la Semiótica en el ámbito latinoamericano, incluidas España y Portugal. Esta iniciativa recibió el respaldo unánime de la Asamblea de la Federación Latinoamericana de Semiótica (FELS) reunida en Guadalajara, México, en julio de 1997. La primera ELSE se realizó en 1997 y se dictaron cursos sobre Semiótica Visual, Semiótica de Peirce y Semiótica del Cine. Un comité organizador está trabajando ya en la organización de la II Escuela Latinoamericana de Semiótica ${ }^{10}$, a realizarse del 26 al 31 de octubre de 1998, en Maracaibo.

La segunda iniciativa aprobada por la AVS fue la creación de la Colección Monográfica de Semiótica Latinoamericana, suerte de anuario que dedicará cada número a un área específica de la Semiótica y que abordará temas latinoamericanos. El primer número deberá publicarse en Noviembre de 1998 y estará dedicado a la Semiótica Visual. Ambas iniciativas se desarrollan en conjunto con el Laboratorio de Investigaciones Semióticas y Antropológicas de la Universidad del Zulia.

${ }^{10}$ Los interesados en participar en los cursos que organiza la Escuela Latinoamericana de Semiótica pueden comunicarse a la siguiente dirección electrónica: jefinol@ven.net o a la siguiente dirección postal: Universidad del Zulia, Facultad de Ciencias, Laboratorio de Investigaciones Semióticas y Antropológicas, Apartado 526 Maracaibo, Venezuela. 


\subsection{Grupos de investigación}

En 1984, gracias a la iniciativa de la Dra. Teresa Espar, se fundó en la Universidad de los Andes, el Grupo de Investigaciones Semiolingüísticas (GIS), el cual desarrolla actividades de investigación en semiótica de la literatura, sociosemiótica, semiótica del discurso jurídico y didáctico. Éste ha sido uno de los grupos más permanentes y activos de la Semiótica venezolana y ha generado un número importante de publicaciones ${ }^{11}$, algunas de las cuales han recibido reconocimientos académicos. En 1985 se fundó en esa universidad la primera cátedra de Teoría Semiótica Sistemática y se realizaron las primeras tesis en el área. En 1990 el GIS fue reconocido oficialmente como grupo de investigación con apoyo presupuestario por parte de los entes financieros universitarios. Este grupo trabaja vinculado al Centro de Investigaciones y Atención Linguiística y al programa de Maestría en Lingüística de la Universidad de los Andes.

También en la Universidad de los Andes, en 1997, gracias a la iniciativa de Rocco Mangieri, se creó la Asociación Venezolana de Semiótica Visual ${ }^{12}$ (AVSV), que reúne a un nutrido grupo de jóvenes investigadores en distintas áreas de la Semiótica Visual y del Arte. La AVSV es una iniciativa surgida de las experiencias del Seminario de Semiótica de las Artes que desde 1994 se desarrolla en el marco de la dirección de postgrado de la Facultad de Arquitectura de la Universidad de los Andes.

Igualmente, el 26 de abril de 1997 se creó en la Facultad de Ciencias de la Universidad del Zulia el Laboratorio de Investigaciones Semióticas y Antropológicas (LISA), el cual reúne a varios investigadores y estudiantes que realizan actividades de investigación en diversas áreas de esa disciplina. En dicho laboratorio participan investigadores de las facultades de Ciencias, Humanidades y Educación (Nancy Torres) y Ciencias Jurídicas y Políticas (Emperatriz Arreaza). Las áreas principales de investigación son la etnosemiótica, en particular trabajos diversos sobre la cultura funeraria venezolana, y la semiótica de la cultura popular (graffiti, discurso publicitario, semiótica del

11 Una de las más importantes publicaciones es la reciente aparición del libro Semiótica general y el discurso literario latinoamericano, de Teresa Espar (1998).

12 El Consejo Directivo de la AVSV está constituido por Rocco Mangieri, Franklin Cañizales, Merysol León, Carlos Pérez, Ondina Briceño, Manuel López, Frank Baiz y Jean Louis Rebillou. 
nombre). También se han desarrollado trabajos sobre el discurso audiovisual (telenovela, cine) y sobre la semiótica del juego y del azar.

En cuanto a la enseñanza de la Semiótica en este período, se debe señalar que se han abierto cátedras de semiótica en el postgrado, especialmente en las maestrías de Lingüística, Antropología, Literatura y Comunicación Social.

\subsection{La semiótica de Peirce}

La semiótica basada en las teorías de Charles Sanders Peirce ha tenido hasta ahora poco eco entre los investigadores venezolanos, e incluso la difusión bibliográfica de su obra ha sido relativamente escasa en el ámbito del país, sobre todo si se le compara con la difusión de los trabajos de otras escuelas europeas. El libro La ciencia de la Semiótica de Charles Sanders Peirce fue editado en 1974 por Nueva Visión, en Argentina. En él se recoge una selección de trabajos del eminente polígrafo norteamericano. El libro de Charles Morris, Fundamentos de la Teoría de los Signos, que había sido publicado por Mouton en 1971, es editado en español por Paidós en 1985. A pesar de estas ediciones tempranas y de algunos trabajos realizados en el ámbito venezolano sobre Peirce, las teorías de estos autores son, lamentablemente, muy poco conocidas en Venezuela.

Recientemente el profesor Aquiles Estée finalizó su doctorado en Semiótica en la Pontificia Universidad Católica de Sao Paulo, programa que dirige la Dra. Lucia Santaella y que está orientado hacia una semiótica peirceana. También en 1998, en el marco de la I Escuela Latinoamericana de Semiótica, en Maracaibo, el Dr. Floyd Merrell dictó un curso de una semana sobre las teorías peirceanas. Igualmente, de 1991 a 1993, José Enrique Finol tuvo la oportunidad de realizar un post-doctorado en el Research Center for Language and Semiotic Studies de la Universidad de Indiana, centro dirigido hasta entonces por el Dr. Thomas Sebeok, uno de los grandes seguidores de las teorías de Peirce.

Visto el auge tomado por las teorías de Peirce en todo el mundo, es posible profetizar que se iniciarán en Venezuela, gracias a un mejor conocimiento de las teorías de ese autor, programas de docencia e investigación que se apoyen en ellas. 


\section{PUBLICACIONES}

Aunque no hay publicaciones especializadas sólo en este campo, existen, no obstante, varias revistas interdisciplinarias en las cuales los resultados de investigaciones semióticas son expuestos regularmente. Una de las primeras publicaciones venezolanas que concentró la mayor parte de sus artículos en el área de Semiótica fue la revista Video-Forum, consagrada a las «ciencias y artes de la comunicación audiovisual», editada dos veces al año por la Academia Nacional de Ciencias y Artes del Cine y la Televisión. La revista apareció por primera vez en noviembre de 1978 y sus primeros números consagraron numerosos artículos al análisis semiótico de temas audiovisuales, con especial énfasis en la telenovela ${ }^{13}$.

Otra publicación que lamentablemente no tuvo la continuidad deseada fue Intertexto, Revista de Semiótica y Psicoanálisis, dirigida por el psicoanalista Hugo McCormick, en Barquisimeto. Ella ofreció durante su aparición un escenario interesante de discusión y análisis de aspectos importantes de la cultura venezolana ${ }^{14}$.

La revista Comunicación es quizás la revista del área de mayor continuidad, permanencia y calidad que existe en Venezuela. Fundada por los sacerdotes jesuitas del Centro Gumilla, en Caracas, esta revista está dedicada fundamentalmente al campo de la sociología y la teoría de la comunicación. No obstante, en sus páginas se han publicado numerosos trabajos del área de la Semiótica.

Otras revistas como Escritura, especializada en la crítica literaria, y Órbita, centrada en el análisis de la comunicación social, han contribuido sensiblemente a la divulgación de temas y análisis vinculados a

${ }^{13}$ Entre esos trabajos publicados en Video-Forum estimo que es pertinente mencionar, en particular, «Para una aproximación semiológica a la novela», de Óscar Moraña, y «La radionovela. Una semiosis entre el pecado y la redención», de Manuel Bermúdez.

14 Intertexto estuvo precedida en Barquisimeto por la publicación La Letra Continua, editada por Fundacultura. El número cero de Intertexto, publicado en 1980 (?), trae artículos de Hugo Bleichmar («Del enunciado a la enunciación, el yo ideal y el ideal del yo: efecto de dos tipos de discurso»), Alberto Conte («La verdad como pre-texto»), José Sazbón («Lenguaje y Escritura»), Víctor Bravo («De la poética linguiística a la semiótica poética») y Hugo McCormick («La neurosis como proceso de (psico)semiosis»). También una extensa entrevista a Eliseo Verón hecha por J.E. Finol, quien había publicado anteriormente una entrevista a Ferrucio Rosi-Landi en El Pez Fumón, 4, julio (1976), Maracaibo: Universidad del Zulia. 
nuestra disciplina y muchos de los semióticos venezolanos han encontrado en ellas un medio eficaz de divulgación científica. También recientemente se ha iniciado la publicación de la revista Omnia, en la Universidad del Zulia, la cual recoge, entre otros, trabajos de investigación vinculados al área semiótica.

Hoy una de las más importantes revistas es probablemente la revista arbitrada Opción editada en la Universidad del Zulia, en Maracaibo, y que se publica con ejemplar regularidad tres veces por año. Hasta ahora han aparecido 24 números que recogen artículos en español, inglés y francés, que son justamente los tres idiomas oficiales de la Asociación Internacional de Semiótica ${ }^{15}$. Varios reconocidos semióticos del mundo han publicado ya en Opción, entre ellos, Eric Landowski, Howard Smith, James J. Liszka, Fernando Andacht, Luisa Ruiz Moreno, Teresa Espar, además del grupo de semióticos zulianos.

En la Universidad de los Andes también se han comenzado a editar dos revistas muy importantes, donde han aparecido varios trabajos en el área semiótica. Una de ellas es Voz y Escritura, de la cual es responsable el Instituto de Investigaciones Literarias, y la otra es Lengua y Habla, del Centro de Investigaciones y Atención Lingüística. Ambas han publicado varios números con diversos trabajos en semiótica y en lingüística.

\section{LA GENERACIÓN DEL FUTURO}

La Semiótica en Venezuela, en nuestra opinión, tiene por delante varios retos que asumir e innumerables aportes que hacer al conocimiento de los procesos de significación y comunicación. Creemos que algunos de los principales retos tienen que ver con la creación, fortalecimiento y ampliación de los grupos de investigación, células fundamentales del desarrollo científico en cualquier campo del conocimiento. Estimamos asimismo que las asociaciones y las reuniones científicas deben mantenerse y fortalecerse, pues ellas juegan un papel

15 La Asamblea General de la Asociación Internacional de Semiótica realizada en Guadalajara, México, durante el sexto congreso de la asociación, aprobó por mayoría abrumadora, la incorporación del español como tercera lengua oficial de la misma. 
capital en la divulgación, promoción y estímulo al desarrollo de esta disciplina y al papel que ella puede cumplir en el conocimiento de la realidad cultural nacional. Por último, en el mundo abierto en el que hoy vivimos esas tareas deben asumirse con una vocación internacional y, en particular, latinoamericana.

\subsection{Fortalecimiento y multiplicación de los grupos de investigación}

A lo largo de estos casi cuarenta años de actividad semiótica en Venezuela, se han consolidado los estudios de esta disciplina, hasta tal punto que hoy contamos con una generación de relevo que está muy bien preparada y que además es numerosa. Esa generación, preparada al calor de los postgrados venezolanos que tienen un alto contenido de formación semiótica, y de los pregrados donde la disciplina ha encontrado condiciones para su fortalecimiento y crecimiento, garantiza su continuación y enriquecimiento.

Papel más importante aún cumplirán sin duda los grupos de investigación (equipos, seminarios, centros, laboratorios), pues es allí donde realmente se fragua la formación y la pesquisa semióticas. La experiencia en nuestra propia disciplina y en otras disciplinas científicas de mayor tradición, muestra que la existencia de grupos de investigación, constituye un factor definitivo en la consolidación, ampliación y productividad de una disciplina cualquiera. Para que tales grupos de investigación funcionen y permanezcan en el tiempo hay dos condiciones capitales que deben satisfacerse. Por un lado, la presencia de un liderazgo en investigación y docencia, el cual promueve la constitución de un área de interés donde todos participan. Por otro lado, el apoyo institucional que, en el caso de Venezuela viene de dos fuentes principales: las universidades, y en particular sus Consejos de Desarrollo Científico y Humanístico (CDCH), y el Consejo Nacional de Investigaciones Científicas y Tecnológicas (CONICIT) que, junto con las fundaciones regionales de ciencia y tecnología, conocidos en Venezuela como FUNDACITE, pueden contribuir substantivamente al funcionamiento de los grupos de investigación. 


\subsection{Fortalecimiento de las asociaciones $y$ de las reuniones nacionales}

Los avances de 1997, con la reactivación de la Asociación Venezolana de Semiótica y con la realización periódica de los congresos nacionales, deben servir a los semióticos venezolanos para fortalecer el desarrollo de esta disciplina. La activa divulgación de trabajos de investigación nos permitirá mostrarle al país, y en especial a la comunidad científica y académica nacional, los aportes que la teoría semiótica puede hacer al conocimiento de la cultura venezolana, de sus formas de expresión y de sus prácticas significantes. En la medida en que tales aportes se hagan y se divulguen sistemáticamente, en esa misma medida los medios que fomentan la actividad científica prestarán un mayor apoyo a la formación de recursos humanos, en particular a través de la creación de programas de postgrado, una de las metas soñadas por los semióticos venezolanos y que otros países latinoamericanos ya han visto cristalizar.

En esa dirección juega un papel decisivo, complementario de las actividades de los grupos de investigación, la constitución de los capítulos regionales de la Asociación Venezolana de Semiótica (AVS), tarea actualmente en proceso, el mantenimiento y continuidad de los congresos nacionales, así como de las reuniones científicas de las asociaciones llamadas de interés especial, tales como las asociaciones de semiótica visual, las de semiótica del cine y otras. Es responsabilidad fundamental de la Asociación Venezolana de Semiótica organizar los congresos bianuales y promover la constitución de los capítulos regionales.

\subsection{La vocación latinoamericana}

Ahora bien, la semiótica venezolana debe promover una vinculación activa con sus pares latinoamericanos, pues la problemática común, así como la vecindad geográfica y la comunidad lingüística, facilitarian los lazos de cooperación. De ahí que las dos iniciativas tomadas por la AVS, la Escuela Latinoamericana de Semiótica (ELSE) y la Colección Monográfica de Semiótica Latinoamericana, estén orientadas hacia una vocación continental. Aquí debe intervenir la presencia de un lide- 
razgo activo, complementario de la actividad en cada una de las asociaciones en cada país, por parte de la Federación Latinoamericana de Semiótica que hoy preside el Dr. Nicolás Rosa.

Los semióticos latinoamericanos no sólo alcanzamos un logro importante cuando la asamblea general de la Asociación Internacional de Semiótica, reunida en México, aprobó la incorporación del español como tercera lengua oficial de la misma. Pienso que asumimos también un reto cuyo cumplimiento marcará el futuro de la Semiótica en nuestro continente. Me refiero al reto de producir cada año lo suficiente, cuantitativa y cualitativamente, para tener una presencia al menos equiparable a la que se hará en otros idiomas en los congresos internacionales de la asociación ${ }^{16}$.

\section{Referencias Bibliográficas}

Cabeza L., J. (1989). Publicidad y discurso. Maracaibo: Publicaciones de la Facultad de Ciencias de la Universidad del Zulia.

CoqueT, J.-C. et alii (1982). Sémiotique. L'École de Paris. París: Hachette. ESPAR, Teresa (1989). Semiótica general y el discurso literario latinoamericano.

Caracas: Monte Ávila.

RODRÍGUEZ, R. (1988). Resplandor. Caracas: Editorial Larense

SAussure, F. (1965[1945]). Curso de Lingüística General. Buenos Aires:

Losada, S.A.

16 El próximo congreso de la Asociación Internacional de Semiótica se celebrará en Dresden, Alemania, en 1999. 